\title{
Imaging Flash Lidar for Autonomous Safe Landing and Spacecraft Proximity Operation
}

\author{
Farzin Amzajerdian ${ }^{1}$, Vincent E. Roback ${ }^{2}$, Paul F. Brewster ${ }^{3}$, Glenn D. Hines ${ }^{4}$ \\ NASA Langley Research Center, Hampton, Virginia, 23681 \\ Alexander Bulyshev ${ }^{5}$ \\ Analytical Mechanics Associates, Hampton, VA 23666
}

\begin{abstract}
3-D Imaging flash lidar is recognized as a primary candidate sensor for safe precision landing on solar system bodies (Moon, Mars, Jupiter and Saturn moons, etc.), and autonomous rendezvous proximity operations and docking/capture necessary for asteroid sample return and redirect missions, spacecraft docking, satellite servicing, and space debris removal. During the final stages of landing, from about $1 \mathrm{~km}$ to $500 \mathrm{~m}$ above the ground, the flash lidar can generate 3-Dimensional images of the terrain to identify hazardous features such as craters, rocks, and steep slopes. The onboard fli ${ }^{1}$ ght computer can then use the 3-D map of terrain to guide the vehicle to a safe location. As an automated rendezvous and docking sensor, the flash lidar can provide relative range, velocity, and bearing from an approaching spacecraft to another spacecraft or a space station from several kilometers distance. NASA Langley Research Center has developed and demonstrated a flash lidar sensor system capable of generating 16k pixels range images with $7 \mathrm{~cm}$ precision, at a $20 \mathrm{~Hz}$ frame rate, from a maximum slant range of $1800 \mathrm{~m}$ from the target area. This paper describes the lidar instrument design and capabilities as demonstrated by the closed-loop flight tests onboard a rocket-propelled free-flyer vehicle (Morpheus). Then a plan for continued advancement of the flash lidar technology will be explained. This proposed plan is aimed at the development of a common sensor that with a modest design adjustment can meet the needs of both landing and proximity operation and docking applications.
\end{abstract}

\section{Introduction}

The 3-D imaging flash lidar technology is of importance to NASA as it can provide precision and hazard avoidance capabilities for landing missions to the planetary bodies ${ }^{1-4}$ and enable spacecraft autonomous rendezvous and docking with satellites or asteroids ${ }^{5-7}$. When used as landing sensor, the flash lidar can generate 3-D maps of the terrain during the descent phase at several kilometers altitude. By comparing the 3-D maps with onboard terrain maps, the lander's position error is significantly reduced and a divert trajectory towards the pre-designated can be determined. ${ }^{2}$ Once within about a kilometer of the landing site, the high resolution 3-D images of the flash lidar are used to identify hazardous terrain features and the safest landing location at the site. ${ }^{3}$ During final approach, the flash lidar can track terrain features and guide the vehicle to a safe landing location. Flash lidar is a solution for future robotic missions to the Moon and Mars that require landing at pre-designated sites of high scientific value, while avoiding hazardous terrain features, such as escarpments, craters, slopes, and rocks. Future missions planned to pave the path to colonization and mining of the Moon and human landing on Mars will need onboard hazard detection and precision navigation to ensure safe landing near previously deployed assets.

As an autonomous rendezvous proximity and docking or capture sensor, flash lidar can identify the rendezvous target and provide distance and bearing as the vehicle approaches the target satellite or station. The range images of flash lidar will enable new capabilities for resupply missions to the International Space Station, servicing satellites, and space debris

\footnotetext{
${ }^{1}$ ALHAT Sensors Lead, LaRC Remote Sensing Branch, AIAA member

${ }^{2}$ Flash Lidar Lead System Engineer, LaRC Remote Sensing Branch

${ }^{3}$ Flash Lidar Lead Software Engineer, LaRC Flight Software Systems Branch

${ }^{4}$ ALHAT Sensors Electronic Systems Lead, Branch Head, LaRC Remote Sensing Branch

${ }^{5}$ Flash Lidar System Analyst and Algorithms Lead
} 
identification and removal. Flash lidar is also viewed as a critical technology for asteroid mission concepts requiring precision rendezvous, identification of the landing or sampling site location, and precision navigation to the highly dynamic objects that may be tumbling in space. ${ }^{6}$

NASA's interest in flash lidar technology stems from its ability to record full 3-D images with a single laser pulse, freezing the scene on every frame by removing all motion of the transmitter/receiver platform. Unlike earlier topographic imaging lidar systems that generated 3-D images by scanning the laser beam across the scene and measuring the time of arrival for each returned laser pulse, the flash lidar records a full 3-D image frame by illuminating the scene with a single laser pulse and imaging the scene onto one focal plane array (FPA). Each pixel in the FPA takes independent measurements of the lidar pulse time of flight to the target. Therefore, the flash lidar permits much higher frame rates without any blurring or inaccuracies due to platform motion.

The capabilities of flash lidar for landing application was demonstrated by the Autonomous Landing and Hazard Avoidance Technology (ALHAT) project. $^{8-11}$ The flash lidar was demonstrated onboard a rocket-propelled Vertical Testbed (VTB) referred to as Morpheus ${ }^{12}$. The demonstration flights were conducted at a hazard field specifically constructed for this purpose near the north end of the Shuttle Landing Facility (SLF) runway at NASA Kennedy Space Center. The hazard field is a $100 \mathrm{~m} \times 100 \mathrm{~m}$ area simulating a challenging lunar terrain and consists of realistic hazard features (rock piles and craters) and designated landing areas. ${ }^{13}$ Operating in closed-loop with the ALHAT avionics, the flash lidar data was used to select the best safe location within the hazard field and helped the vehicle to execute a divert towards the designated site.

The effectiveness of flash lidar for autonomous rendezvous proximity and docking applications was tested on three Space Shuttle flights before it retired. These demonstration flights to the International Space Station were SpaceX DragonEye in 2009 and 2011 (STS-127 and 133), and the Sensor Test for Orion Rel-Nav Risk Mitigation (STORRM) in 2011 (STS134). ${ }^{14-16}$ In these flights, the ability of the flash lidar to provide relative range and bearing information to the spacecraft was demonstrated.

The flash lidar performance requirements for most operational scenarios being considered for both landing and rendezvous proximity and docking applications are quite similar as described in sections III and IV. This raises the possibility of developing a common flash lidar sensor that can be modified to the specific objectives and accommodation constraints of each mission. However, achieving this vision requires further technology advancement in order to enhance the flash lidar performance beyond the current state of the technology, improve its efficiency and reduce its size and mass, and address its reliable operation in space environment.

\section{Flash Lidar Sensor Overview}

The principal of flash lidar sensor system is illustrated in Fig. 1. The Flash Lidar uses a two dimensional detector array to detect a laser pulse return from the target. The detector's Readout Integrate Circuit (ROIC) measures the laser pulse time of arrival of each individual pixel simultaneously, thus each flash of the laser generates a 3-D image of the target illuminated by the laser beam. In older, more conventional imaging lidar systems, the laser beam is scanned over the targeted area in a raster pattern and a single detector is used to detect consecutive pulses. Thus many laser pulses are required to cover the target area and generate a multi-pixel image with sufficient resolution. For example, generating a 3D map of a $100 \mathrm{~m} \mathrm{X} 100 \mathrm{~m}$ area with $10 \mathrm{~cm}$ resolution (1M pixels) using a typical $10 \mathrm{kHz}$ laser repetition rate will take 100 seconds which is not practical for a landing scenario. Another major challenge of a scanning system is controlling the laser beam pointing from a moving platform and then estimating the laser spot position in the target area for each transmitted pulse. By recording a full 3-D image with a single laser pulse, the Flash Lidar provides a much higher image frame rate, eliminating the need for a fast laser beam scanning

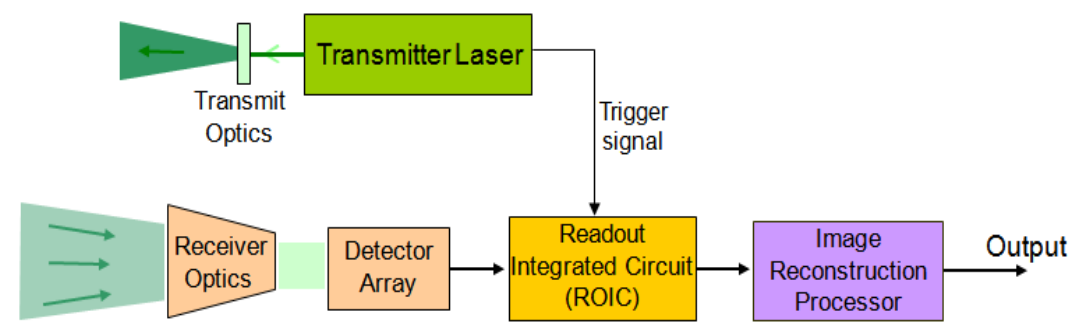

Figure 1. Schematic of Flash Lidar Sensor System. mechanism, and mitigating the effects of platform motion.

The flash lidar developed and demonstrated under the ALHAT project is based on a 3-D sensor engine developed by Advanced Scientific Concepts (ASC) ${ }^{18,19}$. The sensor engine consists of a detector array integrated with a matching ROIC, the associated detector/ROIC power supply, control electronics, and a real-time processor that calibrates the output of the 
ROIC and outputs both range and intensity image frames. The ASC sensor engine has a 128x128 pixel array that is capable of generating range images with $7 \mathrm{~cm}$ precision at up to 30 frames per second. Figure 2 shows the ALHAT flash lidar consisting of a sensor head and an electronics chassis. The sensor head houses the sensor engine, the transmitter laser, and the transmit/receive optics, while the electronics box houses the sensor Controller and Data Handling $(\mathrm{C} \& \mathrm{DH})$ unit, the laser driver, the power conditioning/distribution unit, and temperature control boards. The transmitter laser, developed by Fibertek, generates $50 \mathrm{~mJ}$ pulses at 1.06 micron wavelength. The laser output beam has a uniform square shaped beam matching the detector array. The lidar $\mathrm{C} \& \mathrm{DH}$ unit performs a number of functions including controlling and monitoring various lidar components, interfacing with avionics, and performing image processing and conditioning. Flash lidar is able to detect hazardous terrain features as small as $30 \mathrm{~cm}$ from about $1.8 \mathrm{~km}$ of distance when its field of view is adjusted for $10 \mathrm{~cm}$ spatial resolution.
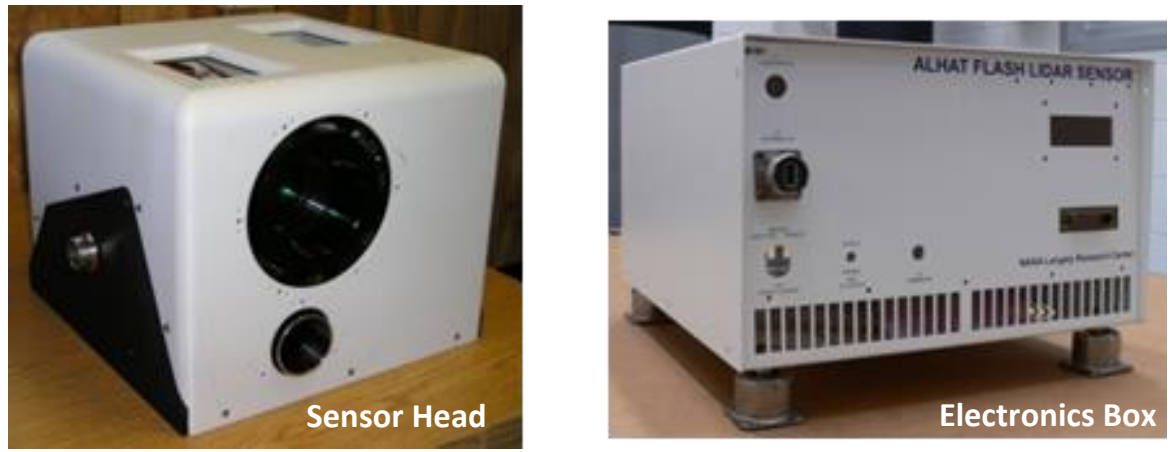

Figure 2. ALHAT Flash Lidar Sensor.

\section{Landing Operational Concepts and Requirements}

As a landing sensor, flash lidar can perform four essential functions during descent and landing phases: Altimetry, Terrain Relative Navigation (TRN), Hazard Detection and Avoidance (HDA), and Hazard Relative Navigation (HRN) ${ }^{2-4}$. Figure 3 illustrates flash lidar operation in the context of a lunar landing. The lidar begins its operation at about $20 \mathrm{~km}$ above the ground after the thruster rocket firing is initiated. ${ }^{20}$ At this stage, the lidar transmitter beam is focused to illuminate only a few pixels in the center of the detector array to measure range to the ground. Reducing the divergence of the lidar transmitter beam to a fraction of its receiver field of view increases its operational slant range to well over $20 \mathrm{~km}$ from a nominal $2 \mathrm{~km}$. The ground-relative altitude measurements provided by the flash lidar reduces the vehicle position error significantly since the Inertial Measurement Unit (IMU) suffers from drastic drift over the travel time from the Earth. The IMU drift error can be over $1 \mathrm{~km}$ for a Moon-bound vehicle and over $10 \mathrm{~km}$ for Mars. Accurate altitude data reduces position error to a few hundred meters. When the altitude drops to about $15 \mathrm{~km}$, the lidar beam is expanded to illuminate approximately a thousand detector pixels. In this phase of its operation, the flash lidar generates relatively low-resolution elevation data of the terrain below which are subsequently compared with stored maps having known surface features such as large craters. This process, referred to as Terrain Relative Navigation, further reduces the vehicle's relative position error from hundreds of meters to tens of meters. From about $1 \mathrm{~km}$ to $0.5 \mathrm{~km}$ altitude, the flash lidar operates with its full field of view, generating a high resolution elevation map of the landing area while identifying hazardous features such as rocks, craters, and steep slopes. This elevation map is then processed to determine the most suitable landing location (HDA function). The flash lidar then continues to update the map in order to establish a trajectory toward the selected landing location. This phase of flash lidar operation is referred to as Hazard Relative Navigation. The flash lidar operation terminates at approximately $100 \mathrm{~m}$ above the ground before the vehicle thrusters create a dust plume.

In order to perform the HDA function at $1 \mathrm{~km}$ altitude, the lidar must have a maximum operational range of $1.4 \mathrm{~km}$ assuming a 45 degrees trajectory angle. Considering that the intensity of the reflected laser light decreases with the incident angle relative to the surface, the maximum operational range of the same lidar at normal incident will be $2 \mathrm{~km}$. Therefore, in the landing scenarios where the trajectory is nearly vertical, such as past Mars landings, the lidar transmitted pulse energy or its receiver aperture can be reduced. In the case of the Mars landing, the altimetry and TRN functions may also be initiated at a lower altitude of about $10 \mathrm{~km}$ when the vehicle velocity is reduced to well below hypersonic speeds by parachutes or deployable decelerators and the heat shield is released. 


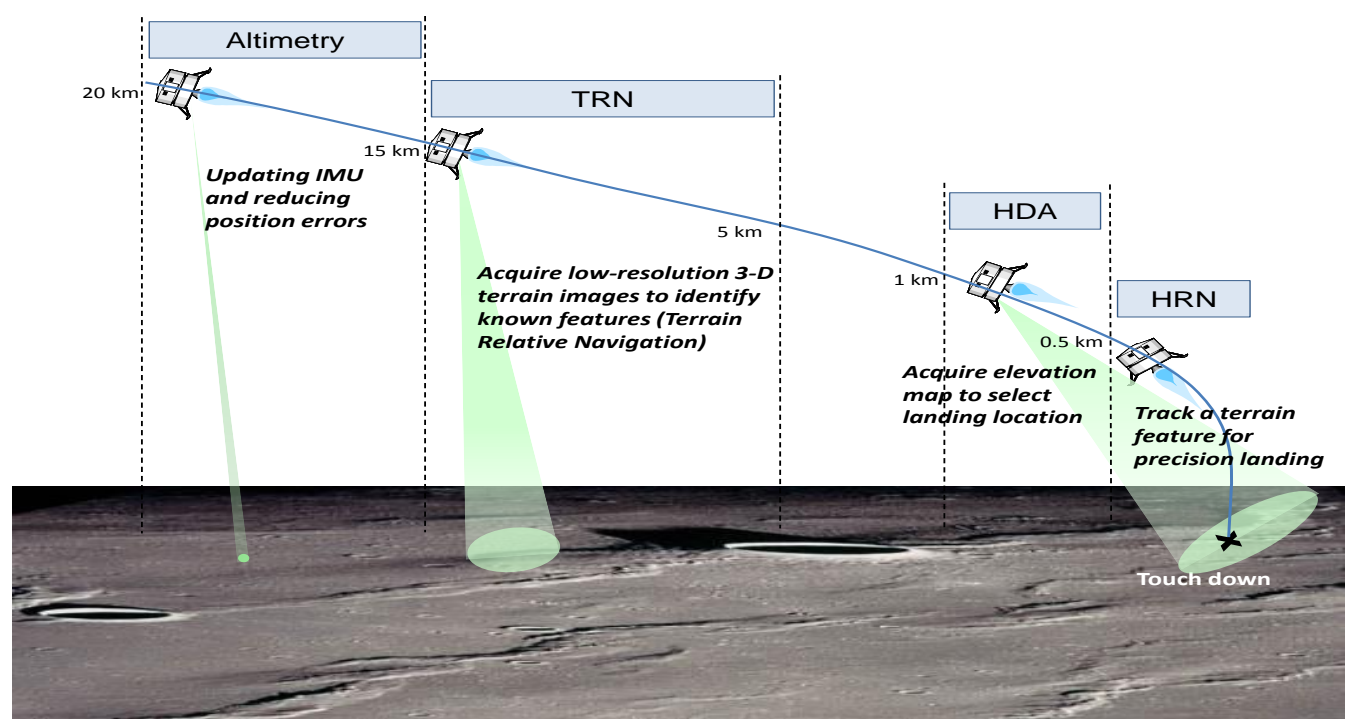

Figure 3. Flash lidar operational concept.

\section{Rendezvous Proximity Operations and Docking}

NASA is interested in developing a common capability for a wide range of missions requiring autonomous Rendezvous Proximity Operations and Docking (RPOD). These missions include human landing on the Moon and Mars, lunar mining, crew and supply transportation to and from the International Space Station, satellite servicing, space debris removal, and asteroid sample return and redirect (Fig. 4). The common capability implies that the system must be: 1) fully autonomous, meaning RPOD can be executed onboard without ground support; and 2) fully operational with both cooperative and noncooperative targets. Non-cooperative targets means no RF transmitters, optical reflectors, or pre-installed distinguishing markings are on the targeted body $\mathrm{f}^{6,7}$. A flash lidar based solution will be a major component of the autonomous RPOD system due to its ability to identify a docking location on the targeted body (either on man-made platforms or planetary bodies) and to provide the necessary bearing, range, and relative attitude data for executing the rendezvous and docking maneuver ${ }^{7}$. The flash lidar operational scenario for RPOD is similar to that of a landing application. The main difference is the size of the target body, which may be smaller than the lidar field of regard for the RPOD application. The flash lidar starts its operation from tens of kilometers away using a few of its pixels to determine the distance to the target. The flash lidar will then operate in full field of view from $2 \mathrm{~km}$ to $3 \mathrm{~km}$ distance to characterize the target surface and identify the docking location. Below $1 \mathrm{~km}$, the flash lidar provides relative range and velocity measurements, along with 3-D range images that are used to compute the vehicle relative pose for use by the navigation filter.

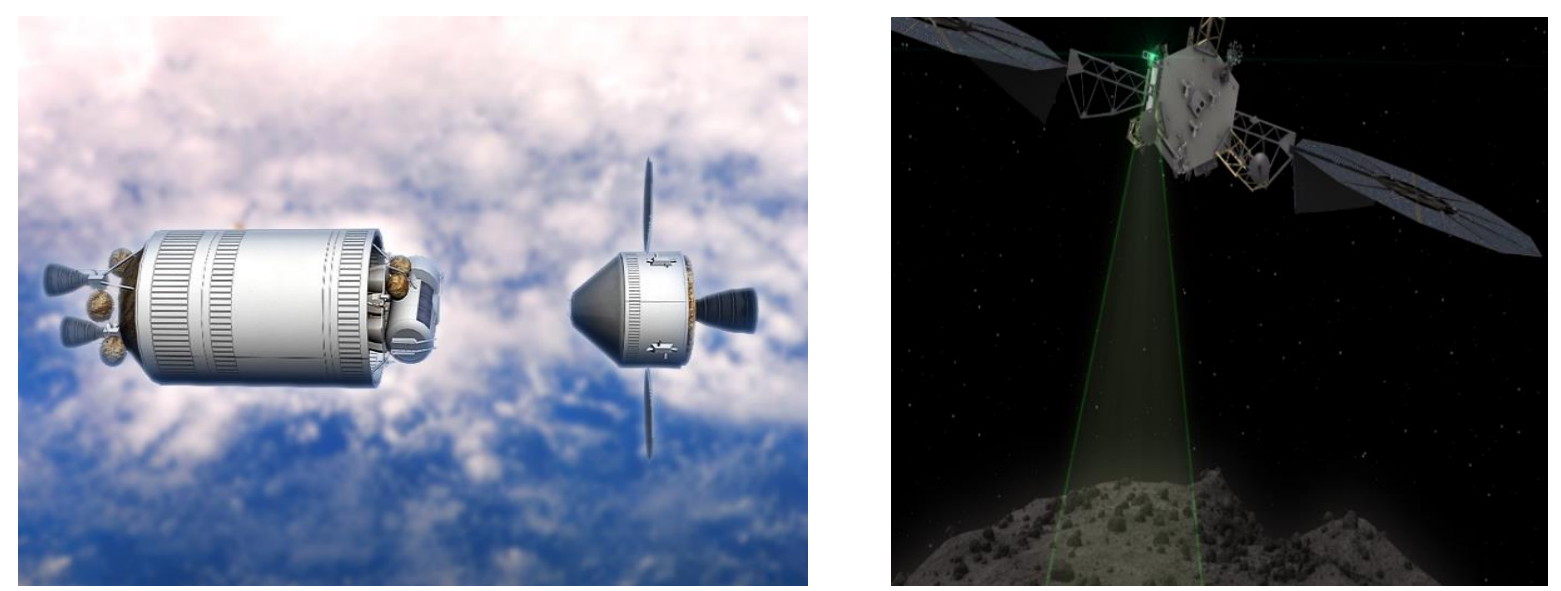

Figure 4. Orion vehicle docking with an Earth Departure Stage (left); Lidar sensor characterizing an asteroid surface before terminal approach for collecting samples or capturing a boulder (right). 


\section{ALHAT Demonstration Flight Tests}

The operation of the flash lidar was characterized during different stages of its development that included extensive laboratory experiments, dynamic truck tests, and three helicopter and one fixed-wing aircraft flight test campaigns. ${ }^{21-25}$ These tests helped the development of the prototype unit and functional demonstration onboard the rocket-powered Morpheus vehicle (Fig. 5). The flight tests also included a Navigation Doppler Lidar ${ }^{17,26,27}$ and a long range laser altimeter ${ }^{17,28}$ that we developed under the ALHAT project along with the flash lidar. The prototype lidars were fully integrated with other ALHAT subsystems ${ }^{8,20}$ that included Hazard Detection System (HDS) ${ }^{9}$, developed by NASA-JPL, and Autonomous Navigation System (ANS) ${ }^{9,20}$, built by NASA-JSC. The HDS uses the flash lidar images to generate a digital elevation map of the landing area and to subsequently select the best landing location given the vehicle's constraints and the mission objectives. The ANS uses the velocity and altitude data from the Doppler Lidar to precisely determine the vehicle position and navigate the vehicle to the safe landing site provided by HDS.

Figure 5 shows the flash lidar along with the Doppler lidar and laser altimeter on the Morpheus vehicle. Morpheus was built by NASA Johnson Space Center (JSC) to demonstrate advanced propulsion and GN\&C technologies for future landing missions. ${ }^{12}$ Initially a series of integration tests were conducted to validate sensor interfaces and operational procedures. ${ }^{25}$ These integration tests included three tethered tests during which the lidars were activated to communicate and provide data to various avionics systems while the Morpheus vehicle was suspended from a crane and executed a controlled flight procedure. After successful tether tests, Morpheus flight tests were conducted at the hazard field specifically constructed for this purpose near the north end of the Shuttle Landing Facility (SLF) runway at NASA Kennedy Space Center (Fig. 6). The hazard field is a $100 \mathrm{~m} \mathrm{x} 100 \mathrm{~m}$ area simulating a challenging lunar terrain and consists of realistic hazard features (rock piles and craters) and designated landing areas. ${ }^{13}$
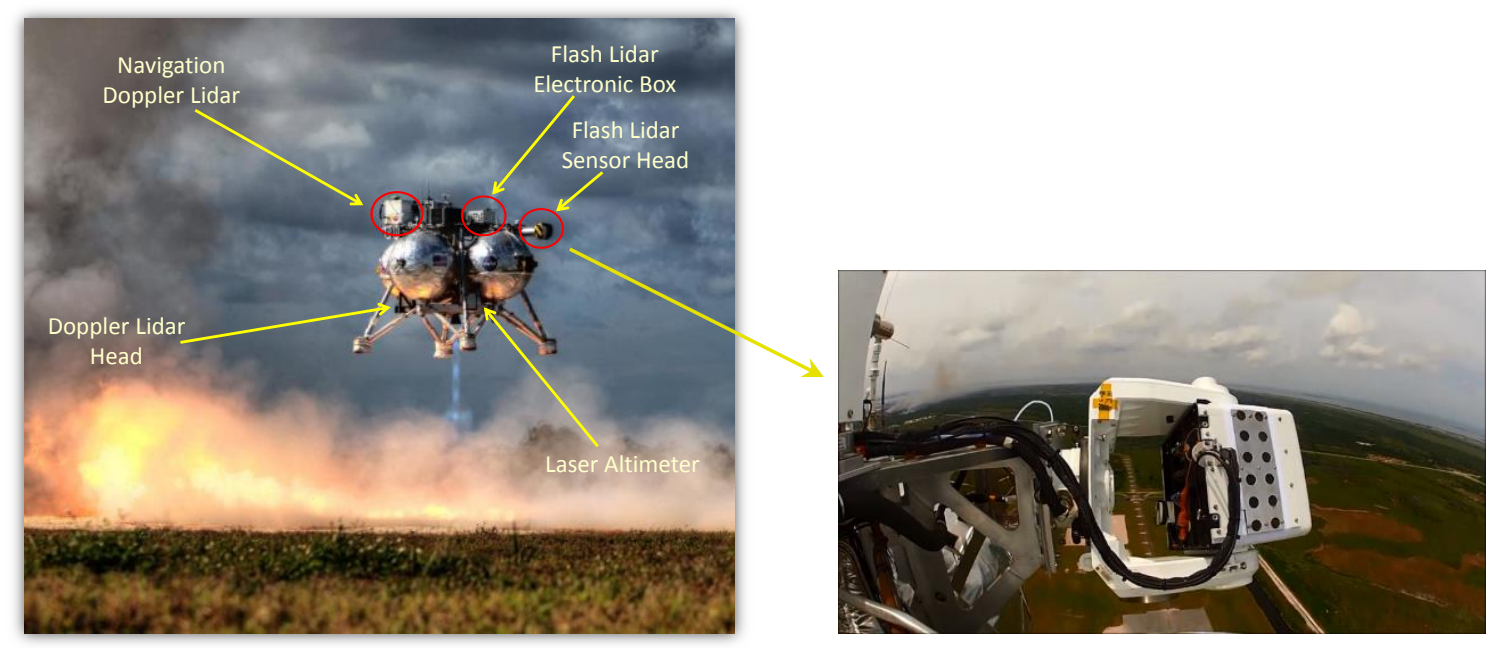

Figure 5. Lidar sensors integrated onto rocket-propelled Morpheus vehicle.

The flight profile, as shown in Fig. 6, is designed to demonstrate the autonomous safe landing system that controls the vehicle flight trajectory to the selected safe site and executes a landing maneuver using the lidar sensors' data. Morpheus launches from a pad next to the SLF runway and climbs to $250 \mathrm{~m}$ and then travels toward the hazard field about $500 \mathrm{~m}$ downrange. A few seconds after the vehicle begins its descend trajectory, the flash lidar maps the hazard field and provides it to the HDS to identify the safe landing locations and selects the best one. Once the coordinates of the landing location are provided to the navigation system, the vehicle uses the Doppler Lidar to precisely navigate to the selected location within the hazard field.

As shown in Fig. 5, the flash lidar head is mounted to a 2-axis gimbal to point the lidar at the hazard field (targeted landing area) and to execute a raster scan pattern that allows mapping the whole field. The lidar receiver field-Of-View (FOV) was chosen to be $1.0 \mathrm{deg}$. to allow for $10 \mathrm{~cm}$ Ground Sample Distance (GSD) from a $750 \mathrm{~m}$ slant range to the target site. Prior analysis indicated that a maximum GSD of $10 \mathrm{~cm}$ is required for reliable detection of hazards of $30 \mathrm{~cm}$ in dimensions, given a lidar range precision of $7 \mathrm{~cm} .{ }^{3}$ The gimbal, controlled by the HDS using the vehicle position and attitude, ensured a series of overlapping lidar image frames that can be stitched together to create a $100 \mathrm{~m} \times 100 \mathrm{~m}$ Digital Elevation Map (DEM). About one hundred lidar flashes is required to produce a $100 \mathrm{~m}$ x $100 \mathrm{~m}$ DEM with the current $128 \times 128$ pixels lidar. All the processing of the flash lidar range images were performed, close to real-time, onboard 
Morpheus by the HDS to generate a DEM, identify the safe landing locations, select the most suitable site, and provide its coordinate to the navigation system. Fig. 7 shows two examples of the flash lidar data from the Morpheus flights. One is a range image from a slant path angle of $30^{\circ}\left(60^{\circ}\right.$ angle of incidence for the lidar) showing some rock piles and the ground slope (color gradient from bottom to top) as apparent from the lidar view angle. The second is a derived elevation image showing rocks as small as $30 \mathrm{~cm} .^{11}$

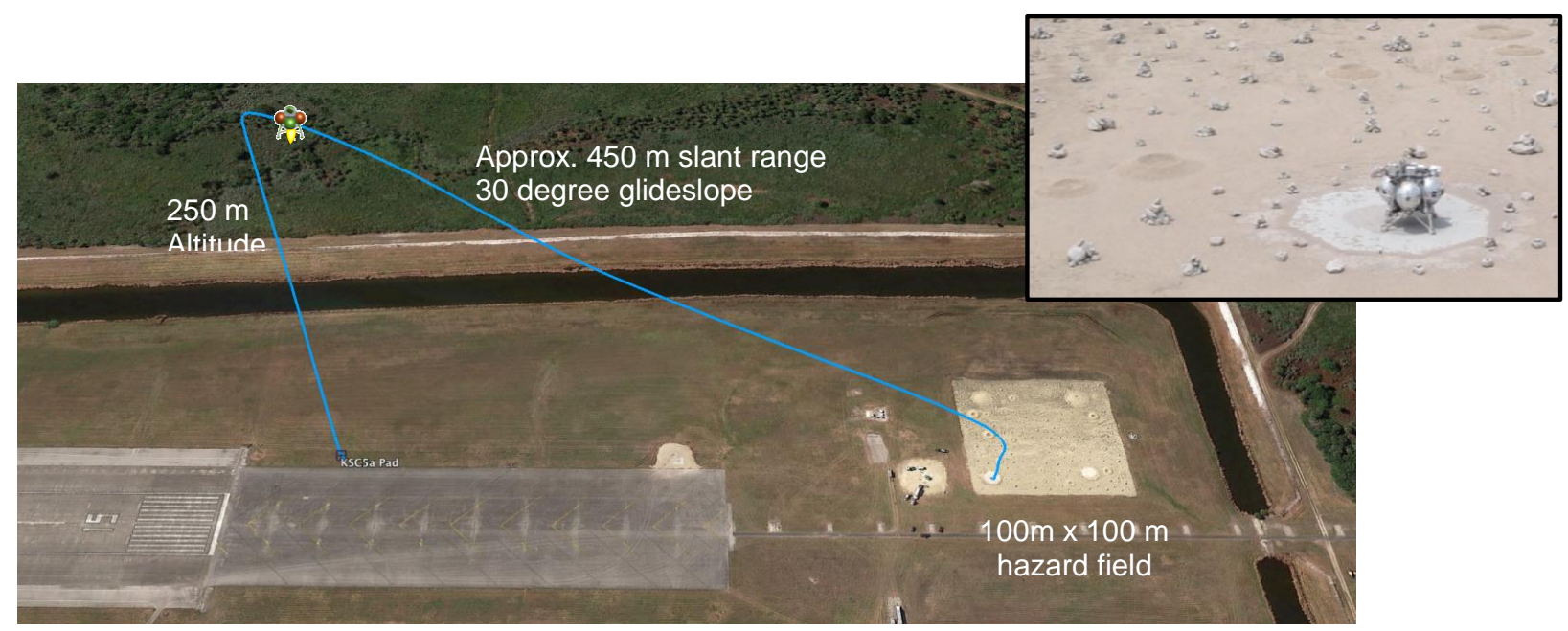

Figure 6. Flight profile for demonstrating autonomous safe landing at a hazard field (simulated lunar terrain) constructed at north end of Shuttle Landing Facility.
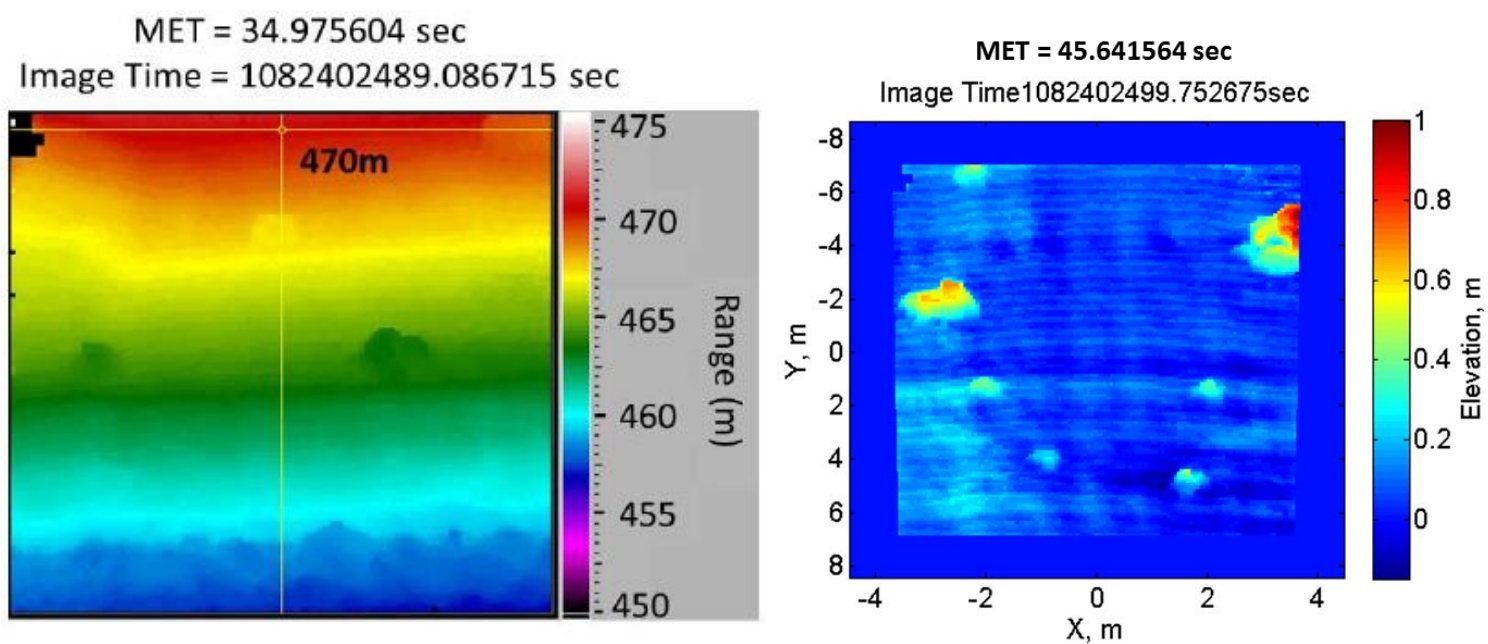

Figure 7. Examples of flash lidar data from Morpheus flight test. Range image on the left shows some rock piles and the ground slope (color gradient) as seen by the lidar, and the image on the right is a projected elevation image showing rocks as small as $30 \mathrm{~cm}$.

\section{Flash Lidar Technology Advancements}

The Morpheus flights proved to be an excellent demonstration of the flash lidar capabilities for future landing missions. ${ }^{9-11}$ However, further technology advancement is required for the development of a multi-purpose flash lidar that can fully meet the needs of a wide range of landing missions as well as the rendezvous and capture missions. Such a common multi-purpose 3-D imaging sensor will present a significant cost saving and risk reduction for a number of missions such as robotic landings on the Moon, Venus, and Saturn moons, human precursor missions to Mars, asteroid redirect and sample return, transport missions to the international space station, and satellite servicing missions. The flash lidar technology advancement and maturation includes performance enhancement, design optimization for size and power reduction, and improved robustness and space qualification considerations. Table 2 lists the current ALHAT flash lidar 
specifications and the desired parameters of a multi-purpose sensor instrument. A major driver in establishing the flash lidar advancement path is the need to map a $100 \mathrm{~m}$ x $100 \mathrm{~m}$ area with $10 \mathrm{~cm}$ resolution (GSD) that is a 1 M pixels digital elevation map (DEM), but a flash lidar with a $1 \mathrm{M}$ pixels detector array may be impractical for foreseeable future. This type of high-resolution sensor will require significant advancements in Avalanche Photodetector (APD) array and the associated Readout Integrated Circuit (ROIC) chip. In addition to almost two orders of magnitude larger number of pixels, the hybridized detector array and ROIC must have achieve a considerably smaller pitch size and much lower noise and higher gain than the current state of technology in order to allow for a reasonable laser pulse energy and receiver aperture size. A near-term compromise could be a 100k-class detector array and use of multiple image frames to construct a $1 \mathrm{M}$ pixels DEM. There are two possible approaches for generating a DEM larger than the individual flash lidar images. The first approach is scanning the lidar field of view (FOV) over the target area to generate a mosaic of image frames that can be stitched together to produce a $1 \mathrm{M}$ pixels DEM. This approach was successfully implemented and demonstrated by ALHAT in Morpheus flight tests using the current $16 \mathrm{~K}$ pixels lidar. The second approach is a Super-Resolution (SR) technique for which the lidar FOV is enlarged to cover the whole area and then a sequence of image frames of the same scene are blended to achieve the desired resolution. Compared with mosaicking, the SR technique has the advantage of requiring a smaller gimbal (since it's only used for pointing), improved DEM quality (lower noise and eliminated bad pixels), and reduced acquisition time (fewer image frames). In addition, the SR algorithm provides independent relative position and orientation data that may help with precision navigation during the final approach phase of landing and RPOD maneuvers.

Table 1. Flash lidar specifications satisfying both landing and RPOD applications compared with current instrument.

\begin{tabular}{|l|c|c|}
\hline Parameter & ALHAT Flash Lidar & Next Generation Lidar \\
\hline Detector Array Size & $16 \mathrm{~K}$ & $>65 \mathrm{k}$ \\
\hline Range Precision within a frame $(1-\sigma)$ & $7 \mathrm{~cm}$ & $3 \mathrm{~cm}$ \\
\hline Frame Rate & $20 \mathrm{~Hz}$ & $20 \mathrm{~Hz}$ \\
\hline Operational Wavelength & 1.06 micron & Eye-safe 1.57 micron \\
\hline $\begin{array}{l}\text { Max Operational Range (for diffuse target } \\
\text { with 30\% reflectivity at normal look angle) }\end{array}$ & $1800 \mathrm{~m}$ & $3000 \mathrm{~m}$ \\
\hline
\end{tabular}

\section{Development of High Resolution Flash Lidar}

As described above, the ALHAT lidar is capable of producing 3-D image frames of distant "non-cooperative' targets with 16k pixels resolution while the STORRM lidar can generate 65k pixels images from distant "cooperative" targets. There is a consensus within the landing and RPOD communities for near-term development of a flash lidar capable of producing 65k pixels range images of distant non-cooperative targets. The higher number of pixels must be accompanied by increased detector array sensitivity so that a reasonable size laser and receiver aperture can be used. The ALHAT project made a serious attempt in developing low-noise, large format detector arrays and associated Readout Integrated Circuit (ROIC) hybridized into a single chip. The ALHAT project supported parallel efforts at Raytheon and ASC to demonstrate the feasibility of 256x256 focal plane arrays using avalanche photodiode (APD) detectors. APD is a highly sensitive semiconductor device that exhibits an internal gain when subjected to relatively high reverse-bias voltage (10s of volts). ASC approach was a scaled version of the InGaAs focal plane used in ALHAT demonstration with some design enhancement for higher sensitivity and range resolution. While Raytheon's approach was based on $\mathrm{HgCdTe}$ detector technology and a silicon-based ROIC concept (Fig. 8). ${ }^{29,30}$ The same Raytheon ROIC technology was used by the STORRM (Sensor Test for Orion RelNav Risk Mitigation) project aboard the Space Shuttle before its retirement. ${ }^{14,31}$ For the STORRM flight the Raytheon ROIC was mated with PIN photodiode array that are roughly an order of magnitude less sensitive than APD arrays but sufficient for imaging cooperative targets such as retro-reflectors.

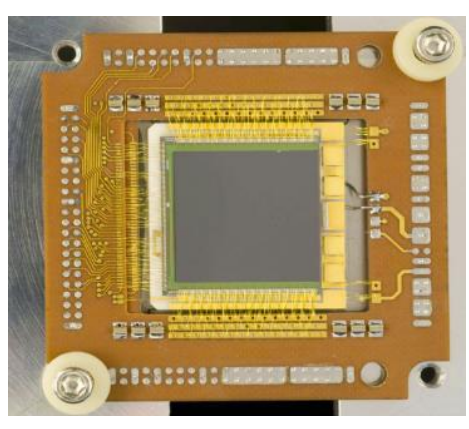

Figure 8. Raytheon 256x256 Sensor Chip Assembly (hybridized detector array and ROIC) delivered in 2012 
The performance simulations and laboratory tests revealed very promising potentials for both Raytheon and ASC approaches. However, limited resources prevented the completion of their development and implementation in actual flash lidar systems. Since then, we have been studying other candidate high density and large area focal plane technologies and their trades for development of a flash lidar sensor that can best meet the needs of both landing and RPOD applications. The focal planes under study include emerging technologies such as single-photon-sensitive Geiger-mode 32 and very high gain and near single-photon-sensitive linear-mode33,34 APDs. Geiger-mode APD is a highly sensitive device responding to a single photon when reverse-biased at a voltage greater than its breakdown voltage. However, a Geiger-mode APD array requires a sequence of laser pulses to generate a 3-D image frame as opposed to linear-mode APD array that can generate a range image with a single but higher energy laser pulse. Therefore, a Geiger-mode flash lidar will require a relatively low pulse energy but high repetition rate laser while a linear-mode lidar requires a low repetition rate laser with moderate pulse energy. The selection of the focal plane array will be based on system level trade studies with consideration of the state of the technology and reliable operation in space environment.

\section{Super Resolution Technique}

The Super-Resolution (SR) technique takes advantage of sub-pixel shifts between multiple, low-resolution images of the same scene to construct a higher resolution image. SR is a well-established technique for enhancing two-dimensional (2-D) images and over the years, and a large number of algorithms have been developed for processing intensity images produced by different types of imaging systems. ${ }^{35,36}$ With the emergence of flash lidar technology, several researchers studied the potential application of the SR technique to this data source. However, these earlier efforts could only achieve 3-D image enhancements when the camera was subjected to a controlled motion within a tightly constrained envelope, with or without image registration. ${ }^{37-40}$ The requirement to tightly constrain camera motion in these techniques precluded their implementation in 3-D cameras installed on many surface, airborne, and space-based platforms since those platforms typically undergo significant excursions in position and orientation. An additional limitation of the past works is that they require external sensors to provide the camera's position and pointing angle. We have developed and demonstrated a 3-D SR algorithm that creates a DEM with a digital magnification factor of four to eight in real-time while providing all six components of the lidar's position and orientation vector. ${ }^{41}$

The SR algorithm utilizes a modified back-projection method and an iteration process to reconstruct a 3-D surface for an arbitrary look-angle. The algorithm calculates the six-degree-of-freedom relative state vector (lidar instrument position coordinates and three components of pointing angle) using consecutive image frames. Determination of the instrument state vector is critical for registration of individual frames prior to combining them to generate a SR image using an inverse filter algorithm. The state vector data provided by this algorithm can also be used by the instrument platform to accurately navigate to the intended destination.

Performance of the SR was analyzed using a high fidelity Mathlab model showing that a digital magnification factor of four to eight (16X to $64 \mathrm{X}$ number of original image pixels) can be achieved by processing 20 consecutive flash lidar frames. The simulations were based on current ALHAT flash lidar characteristics and realistic platform motions. It was also shown that a modest improvement in the flash lidar range noise within the frame and shot-to-shot range precision can provide a digital magnification of eight on a consistent basis using less than 20 frames. The accuracy of the Mathlab model was later verified by actual flash lidar data from a helicopter flight test in a real-time SR processor. ${ }^{41,42}$

The SR algorithm was implemented on two different processing platform: a high-speed Virtex 5 FPGA (FieldProgrammable Gate Array) and a graphics processing unit (GPU). Neither of these SR processors were integrated into the ALHAT flash lidar, but, their operation has been demonstrated by inputting the recoded data from one of the helicopter flights (Field Test 5 campaign, 2012) conducted at NASA-KSC in preparation for Morpheus closed-loop test. ${ }^{43}$ The flight was conducted over the hazard field (Fig. 6) surveyed with $10 \mathrm{~cm}$ spatial and range resolution. Figure 9 shows the truth DEM of the hazard field showing its surface contour, rock piles, and craters. The flight followed a trajectory from $1 \mathrm{~km}$ slant range to $50 \mathrm{~m}$ from the field at a look angle close to 30 degrees. A 5 degree FOV lens was used for this flight to cover a relatively large area of the hazard field.

Figure 10 provides an example of the helicopter flight results obtained from $400 \mathrm{~m}$ slant range. In addition to noise suppression and improving spatial resolution, this example demonstrates another super-resolution attribute, namely the ability to fill in missing data by combining information from several frames. The DEM obtained from a single frame (Fig.10 a) contains a number of dark pixels. These "bad pixels" are basically non-responding detectors. Most of these bad pixels are recovered from other frames as the bad pixels move spatially to adjacent portions of the target, thus exposing the area that they previously blocked. Figure 11 demonstrates how small scale-structures become identifiable and the image noise is reduced. The extended area 2 as shown in Fig. 12 demonstrates that the hazards (rocks) with a diameter $40 \mathrm{~cm}$ and height $20 \mathrm{~cm}$ can be identified. Comparing the true DEM with the restored SR DEM, as illustrated in Figures 11 and 12, 
reveals the merits of the SR image enhancement technique. More detailed description of the SR algorithm, modeling, and test results have been reported in earlier publications. ${ }^{44,45}$

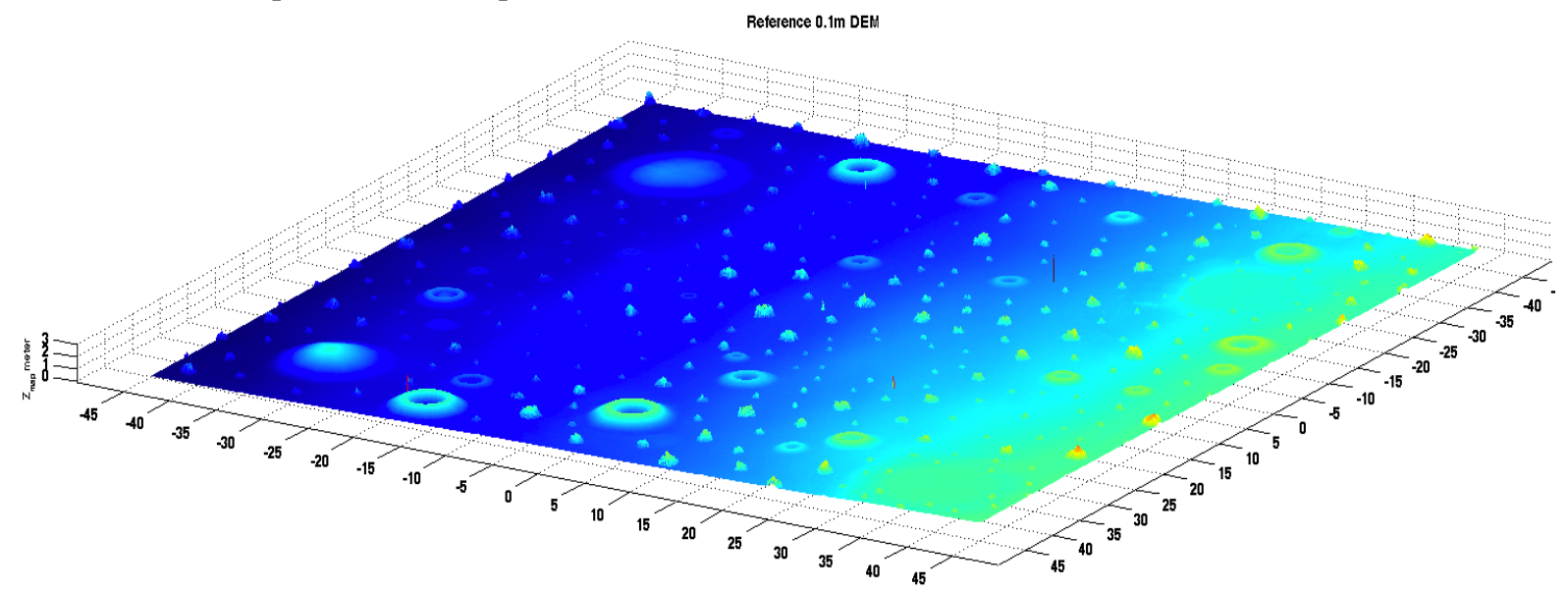

Figure 9. Truth DEM of hazard field with $10 \mathrm{~cm}$ resolution.

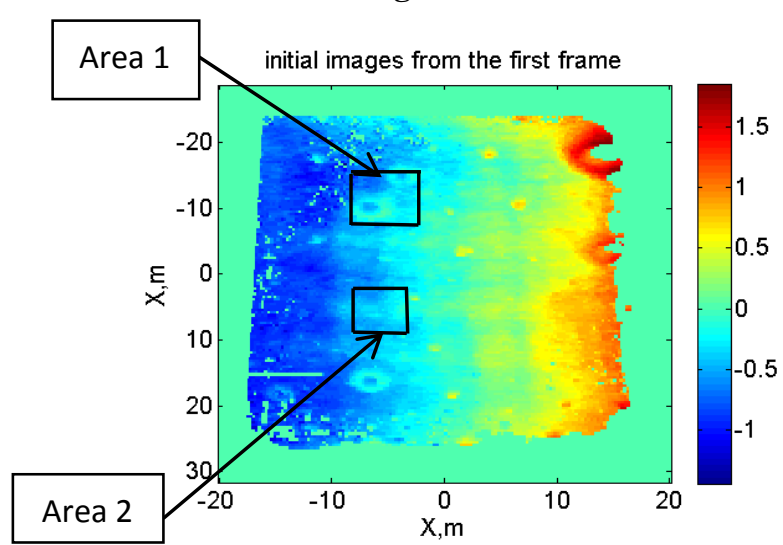

(a)

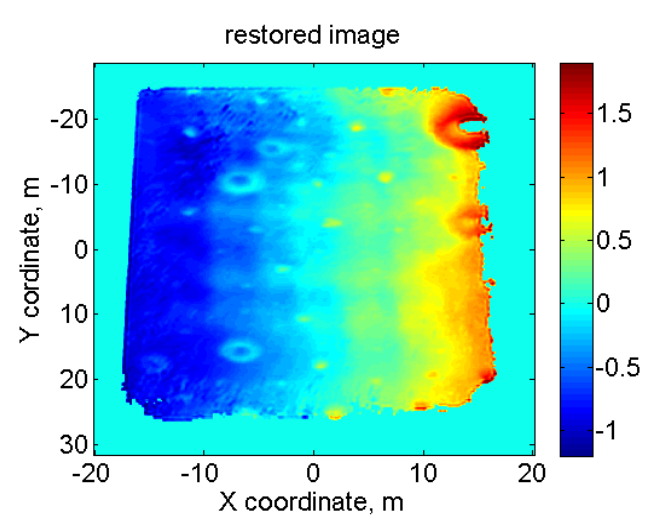

(b)

Figure 10. Results of DEM restoration: a) DEM obtained using 1 frame; b) SR DEM obtained from 20 frames.

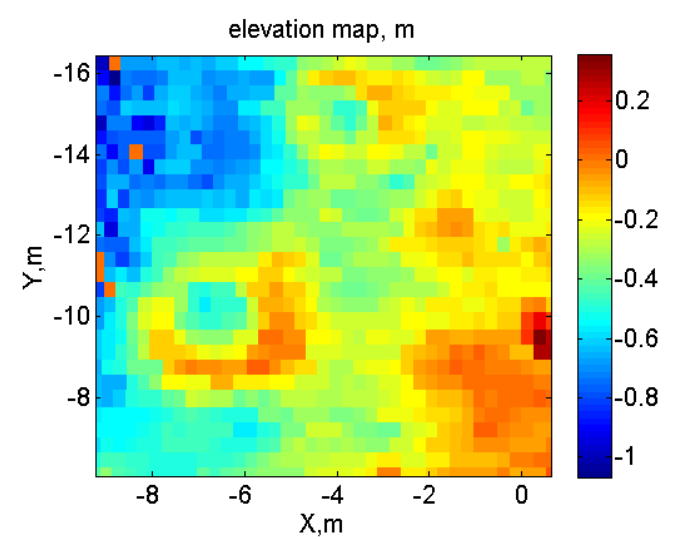

(a)

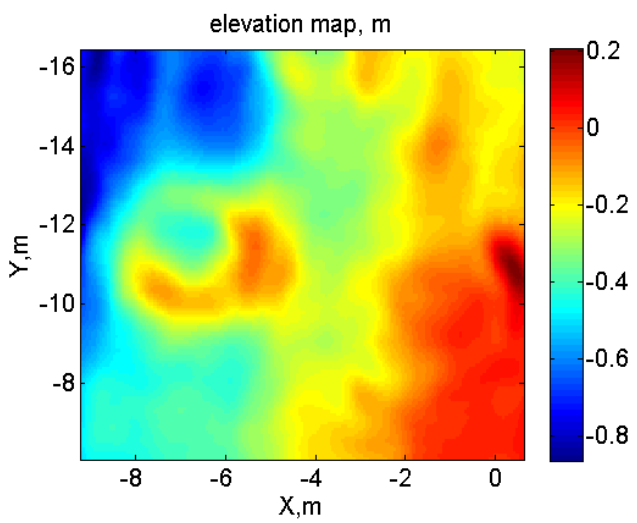

(b)

Figure 11. Zoomed Area 1 (from Figure 10): a) DEM obtained from 1 frame; b) SR DEM obtained from 20 frames. 


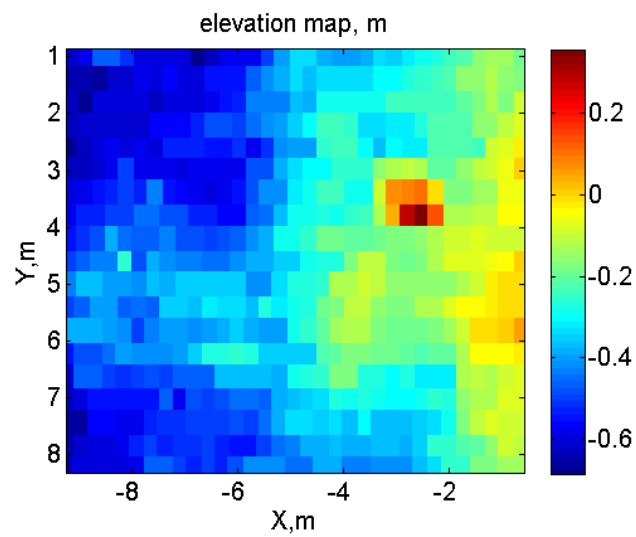

(a)

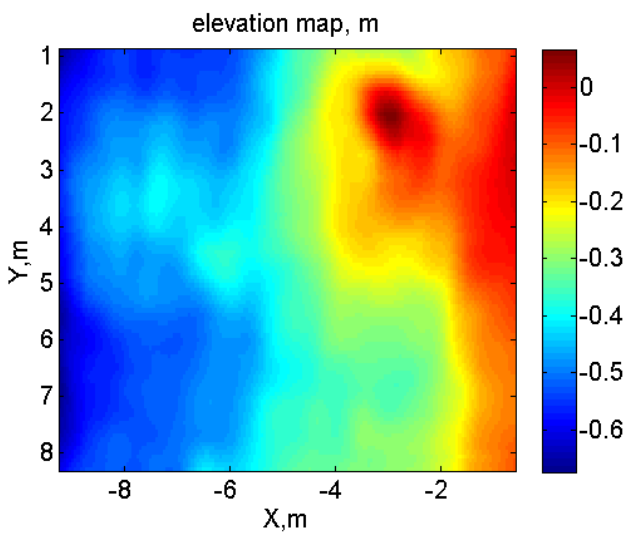

(b)

Figure 12. Zoomed Area 2 (from Figure 10): a) DEM obtained from 1 frame; b) SR DEM obtained from 20 frames.

\section{Conclusion}

Over the past decade, NASA has been actively advancing and testing 3-D flash lidar technology for two distinct applications: autonomous, safe landing on solar system bodies and for autonomous Rendezvous Proximity Operations and Docking. Flash lidar can play a key role in enabling future ambitous robotic and manned missions (Moon, Mars, Jupiter and Saturn moons, etc.) requiring precision landing and onboard hazard avoidance capabilities. Flash lidar is also an important technology for safe and relaibale proximity operation, capture, and docking necessary for asteroid sample return and redirect missions, spacecraft docking, satellite servicing, and space debris removal. The ALHAT project developed and demonstrated a flash lidar sensor system for landing applications through the closed-loop Morpheus flight test campaign. The autonomous rendezvous and docking application of flash lidar was previously assessed by three Space Shuttle missions to the International Space Station. These programs revealed the impact of flash lidar technology on NASA's operation in earth orbit and exploration missions beyond earth orbit. However, the flash lidar in its current state does not fully meet the desired performance specifications for either landing or rendezvous proximity operations and docking applications. A set of common specifications has been defined to direct the flash lidar technology advancement toward a single sensor that satisfies a wide spectrum of landing missions, and for missions requiring autonomous rendzevous and docking capabilities. Such a flash lidar sensor can be developed in the near-term by a combination of an incrementaly larger focal plane array on the order of $100 \mathrm{k}$ pixels and a super-resolution algorithm to produce sufficiently large digital elevation maps with $1 \mathrm{M}$ to $6 \mathrm{M}$ pixels in less than one second from a range on the order of $3 \mathrm{~km}$. Performance of the super-resolution technique and its real-time implementation have been demonstraed through analytical models and simulations and experiemntal data from a helicopter flight test.

\section{Acknowledgments}

The authors are grateful to Chirold Epp, NASA Johnson Space Center, for his guidance and support for eight years as the ALHAT project manager. We also would like to thank Edward Robertson, current ALHAT project manager and NASA's Advanced Exploration Systems (AES) program office for their continued support. The authors also acknowledge the ALHAT and Morpheus team members from the NASA Johnson Space Center and the NASA Jet Propulsion Laboratory for their collaboration and the NASA Kennedy Space Center for facilitating the field tests.

\section{References}

${ }^{1}$ Epp, C. D., Robinson, E. A., and Brady, T., “Autonomous Landing and Hazard Avoidance Technology (ALHAT)”, Proc. of IEEE Aerospace Conference, paper no. 1644, 2008.

2 Johnson, A.E., and Montgomery, J., “An Overview of Terrain Relative Navigation for Precise Lunar Landing,” IEEE Aerospace Conference, 2008.

3 Huertas, A., Johnson, A. E, Werner, R. A., Maddock, R A., "Performance Evaluation of Hazard Detection and Avoidance Algorithms for Safe Lunar Landings,” Proc. IEEE Aerospace Conference, PP 1-20, 2010. 
${ }^{4}$ Amzajerdian, F., Vanek, M. D., Petway, L. B., Pierrottet, D. F., Busch,G. E., Bulyshev, A., "Utilization of 3-D Imaging Flash Lidar Technology for Autonomous Safe Landing on Planetary Bodies, “ SPIE Proceeding Vol. 7608, paper no 80, 2010.

5 Alexandre Pollini, "Flash Optical Sensors for Guidance, Navigation and Control Systems," 35th Annual AAS Guidance and Control Conference, AAS 12-075, 2012

${ }^{6}$ Bo J. Naasz and Michael C. Moreau, "Autonomous RPOD Challenges for the Coming Decade," Proc. of 35th Annual American Astronautical Society Guidance and Control Conference, 2012.

7 B. Barbee, J. Carpenter, S. Heatwole, F. Markley, M. Moreau, B. Naasz, and J. V. Eepoel, "Guidance and Navigation for Rendezvous and Proximity Operations with a Non-Cooperative Spacecraft at Geosynchronous Orbit," Proc. of the AAS George H. Born Symposium, 2010.

8 Epp, C. D., Robertson, E. A., and Carson III, J. M., "Developing Autonomous Precision Landing and Hazard Avoidance Technology from Concept through Flight-Tested Prototypes," Proc. AIAA GN\&C Conference, AIAA 2015-0324, 2015.

9 Carson III, J. M., Robertson, E. A., Trawny, N., and Amzajerdian, F., "Flight Testing ALHAT Precision Landing Technologies Integrated Onboard the Morpheus Rocket Vehicle," Proc. AIAA Space 2015 Conference \& Exposition, Pasadena, CA, 2015.

${ }^{10}$ Nikolas Trawny, Andres Huertas, Michael Luna, Carlos Y. Villalpando, Keith E. Martin, John M. Carson III, Andrew E. Johnson, Carolina Restrepo, Vincent E. Roback, "Flight testing a Real-Time Hazard Detection System for Safe Lunar Landing on the Rocket-powered Morpheus Vehicle," Proc. of AIAA Science and Technology Forum and Exposition, 2015.

${ }^{11}$ Vincent E. Roback, Diego F. Pierrottet, Farzin Amzajerdian, Bruce W. Barnes, Glenn D. Hines, Larry B. Petway, Paul F. Brewster, Kevin S. Kempton, and Alexander E. Bulyshev, "Lidar sensor performance in closed-loop flight testing of the Morpheus rocket-propelled lander to a lunar-like hazard field," Proc. of AIAA Science and Technology Forum and Exposition, 2015.

${ }^{12}$ Olansen, J. B., Munday, S. R., and Devolites, J. L., "Project Morpheus: Lander Technology Development," Proc. AIAA SPACE 2014 Conference \& Exposition, San Diego, CA, 2014.

${ }^{13}$ Rutishauser, D., Epp, C. D., and Robertson, E. A., "Free-Flight Terrestrial Rocket Lander Demonstration for NASA's Autonomous Landing and Hazard Avoidance Technology (ALHAT) System," Proc. of AIAA SPACE, 2012

${ }^{14}$ J. A. Christian, H. Hinkel, C. N. D’Souza, S. Maguire, and M. Patangan, "The Sensor Test for Orion RelNav Risk Mitigation (STORRM) Development Test Objective," Proc. of AIAA Guidance, Navigation, and Control Conference, No. AAS 11-6260, 2011.

${ }^{15}$ John A. Christian, Scott Cryan, "A Survey of LIDAR Technology and its Use in Spacecraft Relative Navigation, "AIAA Guidance, Navigation, and Control Conference, AIAA 2013-4641, 2013.

${ }^{16}$ Space X Press Release, SpaceX'S DragonEye Navigation Sensor Successfully Demonstrated on Space Shuttle, September 29 , 2009

${ }^{17}$ Farzin Amzajerdian, Diego Pierrottet, Larry Petway, Glenn Hines, Vincent Roback and Robert Reisse, "Lidar Sensors for Autonomous Landing and Hazard Avoidance," Proc. of AIAA Space and Astronautics Forum and Exposition, 2013.

${ }^{18}$ Stettner, R., Bailey, H., and Silverman, S., "Three Dimensional Flash Ladar Focal Planes and Time Dependent Imaging," International Symposium on Spectral Sensing Research, Bar Harbor, Maine, 2006.

${ }^{19}$ Stettner, R., "Compact 3D Flash LIDAR video cameras and applications," Proc. of SPIE Vol. 7684, 768405, 2010.

${ }^{20}$ Brady, T., Schwartz, J., and Tillier, C., "System Architecture and Operational Concept for an Autonomous Precision Lunar Landing System," AAS 30th Rocky Mountain Guidance and Control Conference, 2007.

${ }^{21}$ Vincent Roback, Alexander Bulyshev, Farzin Amzajerdian, Paul Brewster, Bruce Barnes, Kevin Kempton, and Robert Reisse, "Helicopter Flight Test of Compact, Real-Time 3-D Flash Lidar for Imaging Hazardous Terrain during Planetary Landing," Proc. of AIAA Space and Astronautics Forum, 10.2514/6.2013-5383, 2013.

${ }^{22}$ Trawny, N., Carson, J. M., Huertas, A., Luna, M. E., Roback, V. E., Johnson, A. E., Martin, K. E., and Villalpando, C. Y., "Helicopter Flight Testing of a Real-Time Hazard Detection System for Safe Lunar Landing," Proc. AIAA SPACE Conference \& Exposition, 2013.

${ }^{23}$ Roback, V. E., Bulyshev, A., Amzajerdian, F., Brewster, P. F., Barnes, B. W., Kempton, K. S., and Reisse, R. E., "Helicopter Flight Test of 3-D Imaging Flash LIDAR Technology for Safe, Autonomous, and Precise Planetary Landing," Proc. of SPIE ,Vol. 8731, SPIE Defense Security, and Sensing Conference, 2013.

${ }^{24}$ A. Bulyshev, D.F. Pierrottet, F. Amzajerdian, G.E. Busch, M. Vanek, and R. Reisse, "Processing of three-dimensional flash lidar terrain images generating from an airborne platform," Proc. SPIE Vol. 7329, 2009.

${ }^{25}$ John M.Carson, Edward A. Robertson, Diego F. Pierrottet, Vincent E. Roback, Nikolas Trawny, Jennifer L. Devolites, Jeremy J. Hart, Jay N. Estes, Gregory S. Gaddis, "Preparation and Integration of ALHAT Precision Landing Technology for Morpheus Flight Testing," Proc. AIAA SPACE Conference and Exposition, 2014.

${ }^{26}$ Pierrottet, D. F., Amzajerdian, F., Petway, L. B., Barnes, B. W., Lockard, G., and Hines, G. D., "Navigation Doppler Lidar Sensor for Precision Altitude and Vector Velocity Measurements Flight Test Results," Proceeding SPIE Vol. 8044, 2011.

${ }^{27}$ Farzin Amzajerdian, Diego Pierrottet, Larry Petway, Glenn Hines, and Bruce Barnes, "Doppler lidar sensor for precision navigation in GPS-deprived environment," Proc. of SPIE vol. 8731, 2013.

${ }^{28}$ Diego F. Pierrottet, Farzin Amzajerdian, Bruce Barnes, "A long-distance laser altimeter for terrain relative navigation and spacecraft landing," Proc. SPIE. 9080, 2014.

${ }^{29}$ W. McKeag, T. Veeder, J. Wang, M.D. Jack, T. Roberts, T. Robinson, J. Neisz, C. Andressen, R. Rinker, T.D. Cook, and F. Amzajerdian, "New Developments in HgCdTe APDs and Ladar Receivers," Proc. SPIE 8012, April, 2011.

${ }^{30}$ Steven Bailey, William McKeag, Jinxue Wang, Michael Jack, and Farzin Amzajerdian, "Advances in HgCdTe APDs and LADAR Receivers," SPIE Proceedings Vol. 7660, 2010. 
${ }^{31}$ Reuben R. Rohrschneider, Jim Masciarelli, Kevin L. Miller, and Carl Weimer, “An Overview of Ball Flash LIDAR and Related Technology Development," AIAA Guidance, Navigation, and Control (GNC) Conference, 2013.

${ }^{32}$ Richard M. Marino, Timothy Stephens, Robert E. Hatch, Joseph L. McLaughlin, James G. Michael E. O'Brien, Gregory S. Rowe, Joseph S. Adams, Luke Skelly, Robert C. Knowlton, Stephen E. Forman, W. R. Davis, “A compact 3D imaging laser radar system using Geiger-mode APD arrays: system and measurements,” Proc. SPIE 5086, 2003.

${ }^{33}$ George M. Williams, David A. Ramirez, Majeed M. Hayat and Andrew S. Huntington, "Time resolved gain and excess noise properties of InGaAs/InAlAs avalanche photodiodes with cascaded discrete gain layer multiplication regions,” J. Appl. Phys. 113, 093705, 2013.

${ }^{34}$ George M. Williams, Madison A. Compton, and Andrew S. Huntington , "Single-photon-sensitive linear-mode APD ladar receiver developments (ROC) Performance of Multi-Gain-Stage," SPIE Proc. Vol 6950 (2008)

${ }^{35}$ S. Park, M. K. Park, and M. Kang, "Superresolution image reconstruction: A technical review," IEEE Signal Processing Magazine 20(3), 21-36, 2003.

${ }^{36}$ S. Young and R. Driggers, "Superresolution image reconstruction from a sequence of aliased imagery," Applied Optics 45(21), 5073-5085, 2006.

${ }^{37}$ G. Rosenbush, T. Hong, and R. Eastman, "Super-resolution enhancement of flash LADAR range data," in Proceedings of SPIE, Unmanned/Unattended Sensors and Sensors Networks IV, 6736, 673614-1 - 673614-10, 2007.

${ }^{38}$ S. Hu, S. S. Young, T. Hong, J. Reynolds, K. Krapels, B. Miller, J. Thomas, and O. Nguyen, "Super resolution for flash LADAR imagery," Applied Optics 49(5),772-780, 2010.

${ }^{39}$ S. Hu, S. Young, T. Hong, J. Reynolds, K. Krapels, B. Miller, J. Thomas, and O. Nguyen, "Super-Resolution for flash LADAR data," Proceedings of SPIE vol. 7300, 2009.

${ }^{40}$ J. Woods, E. Armstrong, W. Armbruster, and R. Richmond, "The application of iterative closest point (ICP) registration to improve 3D terrain mapping estimates using the flash 3D ladar system," Proceedings of SPIE vol. 7684, 2010.

${ }^{41}$ Bulyshev, Alexander; Amzajerdian, Farzin; Roback, Vincent E; Hines, Glenn; Pierrottet, Diego; Reisse, Robert, "Threedimensional super-resolution: theory, modeling, and field test results," Applied Optics, Vol. 53 Issue 12, pp.2583-2594, 2014

${ }^{42}$ Alexander Bulyshev, Farzin Amzajerdian, Eric Roback, Robert Reisse, "A super-resolution algorithm for enhancement of flash lidar data: flight test results," Proc. SPIE Vol. 9020, 2014.

${ }^{43}$ Vincent Roback, Alexander Bulyshev, Farzin Amzajerdian, Paul Brewster, Bruce Barnes, Kevin Kempton, and Robert Reisse, "Helicopter Flight Test of 3-D Imaging Flash LIDAR Technology for Safe, Autonomous, and Precise Planetary Landing," Proc. of SPIE, Vol. 8731, 2013.

${ }^{44}$ Alexander Bulyshev, Farzin Amzajerdian, Eric Roback, Robert Reisse, "A super-resolution algorithm for enhancement of flash lidar data: flight test results," Proc. SPIE Vol. 9020, 2014.

${ }^{45}$ Farzin Amzajerdian, Vincent E. Roback, Alexander E. Bulyshev, Paul F. Brewster, William A. Carrion, Diego F. Pierrottet, Glenn D. Hines, Larry B. Petway, Bruce W. Barnes, and Anna M. Noe, "Imaging flash lidar for safe landing on solar system bodies and spacecraft rendezvous and docking," Proc. SPIE Vol 9465, 2015. 